\title{
Investigation of proNT/NMN secretion from small cell lung carcinoma cells using a mouse xenograft model
}

\author{
KANAKO WAKABAYASHI-NAKAO ${ }^{1}$, KOJI MARUYAMA ${ }^{2}$, HIDEE ISHII ${ }^{2}$, \\ KOJI MURAMATSU ${ }^{3}$, KEIICHI HATAKEYAMA ${ }^{1}$, KEIICHI OHSHIMA ${ }^{1}$, SHUN-ICHIRO OGURA ${ }^{4}$, \\ TAKASHI NAKAJIMA $^{3}$, KEN YAMAGUCHI ${ }^{5}$ and TOHRU MOCHIZUKI ${ }^{1}$ \\ ${ }^{1}$ Medical Genetics Division, ${ }^{2}$ Experimental Animal Facility, Shizuoka Cancer Center Research Institute, Shizuoka; \\ ${ }^{3}$ Pathology Division, Shizuoka Cancer Center Hospital, Shizuoka; ${ }^{4}$ Frontier Research Center, Tokyo Institute \\ of Technology, Kanagawa; ${ }^{5}$ Shizuoka Cancer Center Hospital and Research Institute, Shizuoka, Japan
}

Received March 6, 2012; Accepted May 4, 2012

DOI: 10.3892/or.2012.1926

\begin{abstract}
Proneurotensin/neuromedin N (proNT/NMN), the precursor of neurotensin (NT) and neuromedin N (NMN), is produced by cancer tissues derived from the pancreas and colon. NT stimulates tumor growth and proliferation through its receptors; however, little is known about the precursor molecule in cancer tissues. We previously demonstrated that proNT/NMN is secreted from small cell lung carcinoma (SCLC) cell lines in serum-free conditioned medium, but not from non-small cell lung carcinoma (NSCLC) cell lines. It was suggested that this precursor may serve as a tumor marker for SCLC. In this study, we established in vivo xenograft models to evaluate the possibility of proNT/NMN as a specific tumor marker. SBC3 cells, derived from human SCLC, were inoculated into mice, and the proNT/NMN levels in plasma and tumor tissues were detected using specific antibodies. In contrast to control mouse plasma, the proNT/NMN levels in tumor-bearing mice increased as the tumors grew, and the elevated plasma proNT/NMN levels were decreased by tumor resection. Moreover, proNT/NMN was expressed in SBC3 tumors, suggesting that proNT/NMN was secreted into blood from the tumor, and this secretion may be specific to SCLC.
\end{abstract}

Correspondence to: Dr Tohru Mochizuki, Shizuoka Cancer Center Research Institute, 1007 Shimonagakubo, Nagaizumi-cho, Sunto-gun, Shizuoka 411-8777, Japan

E-mail: t.mochizuki@scchr.jp

Abbreviations: aa, amino acids; CM, conditioned medium; ELISA, enzyme-linked immunosorbent assay; FBS, fetal bovine serum; GRP, gastrin-releasing peptide; HRP, horseradish peroxidase; KLH, keyhole limpet hemocyanin; NMN, neuromedin N; NSCLC, non-small cell lung carcinoma; NT, neurotensin; PC, prohormone convertase; proNT/NMN, proneurotensin/neuromedin N; SCLC, small cell lung carcinoma

Key words: proneurotensin/neuromedin N, neurotensin, small cell lung carcinoma, tumor marker

\section{Introduction}

Proneurotensin/neuromedin N (proNT/NMN) is the common precursor of two neuropeptides, namely neurotensin (NT) and neuromedin $\mathrm{N}(\mathrm{NMN})$. It is expressed in the gut, brain, and adrenals (1), and in the cancer tissues derived from the pancreas (2) and colon (3). In these tissues, proNT/NMN undergoes differential processing, generating different patterns of maturation products such as NT, NMN, large NT, and large NMN (4-8). The biological and pharmacological behaviors of NT have been extensively studied, and NT is known to regulate digestive processes including gastrointestinal motilities and pancreatic and biliary secretions (9). In addition, NT stimulates the growth and proliferation of cancer cells derived from the gut, lungs, pancreas, and prostate through NT receptors (10-12). Friry et al (13) demonstrated that large forms of the processed products are more resistant to degradation than NT and NMN. Therefore, these products might function as longlasting activators of NT receptors. However, little is known about the behavior of its precursor the proNT/NMN.

We previously used MS/MS proteomic analysis to identify secreted proteins produced by lung carcinoma cell lines in serum-free conditioned medium (14). In addition to the proteins previously found in serum-free conditioned media, proNT/NMN was identified in the media from small cell lung carcinoma (SCLC) cell lines, but not from non-small cell lung carcinoma (NSCLC) cell lines. These results suggest that proNT/NMN is specifically secreted from SCLC and might be a potential tumor marker.

To investigate the specific secretion of proNT/NMN from SCLC, we established in vivo xenograft models of SCLC. SBC3 cells, which secrete proNT/NMN mostly in SCLC cell lines, were inoculated into mice, and the plasma proNT/NMN levels of those mice were detected using specific antibodies. ProNT/NMN was detected in the plasma and tumor tissues of SBC3 tumor-bearing mice, while it was not detected in control mice. In addition, plasma proNT/NMN levels had a high correlation with tumor volume. These results suggest that SBC3-derived tumors produce and secrete proNT/NMN into blood. 
A

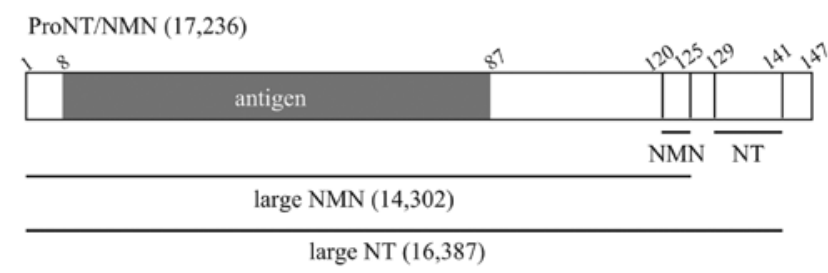

B

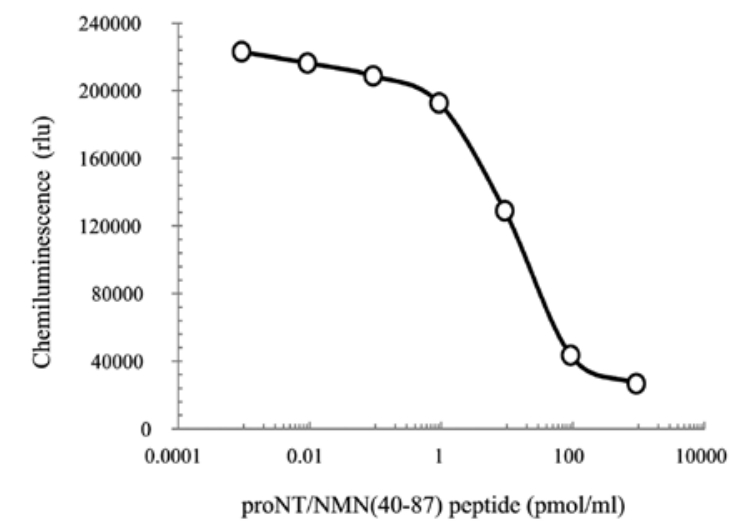

Figure 1. Schematic illustration of proNT/NMN (A) and a standard curve for the proNT/NMN ELISA using proNT/NMN (aa 40-87) (B).

\section{Materials and methods}

Antibodies. We established polyclonal antibodies against the peptide sequences of proNT/NMN (amino acids (aa) 8-87) shown in Fig. 1A. Chemically synthesized proNT/NMN (aa 8-87) was conjugated with keyhole limpet hemocyanin (KLH) and then purified. Antibodies were obtained by immunizing three SPF Japanese white rabbits with KLH-conjugated peptides.

Cell culture and preparation of serum-free conditioned medium. SBC3, a small cell lung carcinoma cell line, was purchased from the Japanese Cancer Research Bank. SBC3 cells were maintained in RPMI-1640 (Sigma, St. Louis, MO, USA) medium supplemented with 10\% FBS (Gibco, Grand Island, NY, USA), penicillin (100 U/ml), and streptomycin $(0.1 \mathrm{mg} / \mathrm{ml})$ in a humidified $5 \% \mathrm{CO}_{2}$ incubator. Serum-free conditioned media was prepared as previously described (14).

Animals. Six-week-old athymic nude mice (BALB/cAJcl$\mathrm{nu} / \mathrm{nu}$ ) were purchased from CLEA Japan (Tokyo, Japan), and NRG mice (NOD.Cg-RagI $I^{\text {tmlMom }} I l 2 \mathrm{rg}^{t \mathrm{tmlWjl}} / \mathrm{SzJ}$ ) were purchased from Charles River (Wilmington, MA, USA). NRG mice were bred in the Experimental Animal Facility of Shizuoka Cancer Center Research Institute, and their offspring was used for experiments. All animal experiments were carried out in accordance with the general guidelines for animal experiments of Shizuoka Cancer Center Research Institute.

In vivo experiments. Nude mice were subcutaneously inoculated with SBC 3 cells $\left(5 \times 10^{6}\right.$ cells in $0.1 \mathrm{ml} \mathrm{PBS} /$ mouse) into the right forelimb, and blood samples were collected once a week. The tumor size was also measured before blood collection to calculate the tumor volume. NRG mice were intradermally inoculated with $\mathrm{SBC} 3$ cells $\left(5 \times 10^{6}\right.$ cells in $0.1 \mathrm{ml} \mathrm{PBS} /$ mouse) to examine the effect of tumor resection, and divided into two groups ( $n=5$ per group). Four weeks after inoculation, tumors were surgically resected from one group and blood was collected from the non-resected and control mice after 24, 48 and $120 \mathrm{~h}$. Blood samles were centrifuged, and plasma was collected. Plasma samples were stored at $-80^{\circ} \mathrm{C}$ until use.

Preparation of mouse tissue lysates. Frozen tumor tissues were treated with lysis buffer containing $7.5 \mathrm{M}$ urea, $2.5 \mathrm{M}$ thiourea, $12.5 \%$ glycerol, $50 \mathrm{mM}$ Tris- $\mathrm{HCl}(\mathrm{pH} 7.4), 2.5 \%$ $\mathrm{N}$-octylglucoside, $6.25 \mathrm{mM}$ Tris-carboxyethyl phosphine hydrocholine (TCEP), and $1.25 \mathrm{mM}$ protease inhibitor, and homogenized by TissueLyser II (Qiagen K.K., Tokyo, Japan). Following rotation for $1 \mathrm{~h}$, the samples were centrifuged at $14,000 \mathrm{x} \mathrm{g}$ for $30 \mathrm{~min}$ at $4^{\circ} \mathrm{C}$, and the supernatants were collected. Protein concentrations were determined by the Bradford assay (Bio-Rad Laboratories, Hercules, CA, USA).

Enzyme-linked immunosorbent assay (ELISA). Microtiter plates (96-well, Nunc C8 Maxisorp Immuno module; Nunc, Roskilde, Denmark) were coated with $100 \mu \mathrm{l}$ of goat antirabbit $\mathrm{IgG}(\mathrm{Fc})$ solution (diluted 1:200 in 0.1 $\mathrm{M} \mathrm{NaHCO}_{3}$; $\mathrm{MP}$ Biomedicals, Irvine, CA, USA) and incubated for $24 \mathrm{~h}$ at $4^{\circ} \mathrm{C}$. After incubation, the antibody solution was removed, and the wells were blocked overnight at $4^{\circ} \mathrm{C}$ by using block ace solution (DS Pharma Biomedical Co., Ltd., Osaka, Japan). Following three washes with $0.05 \%$ Tween-20 in PBS, the biotinylated antigen solution [20 ng/ml biotinyl-proNT/NMN (aa 40-87), prepared in PBS containing $0.1 \%$ BSA], the plasma samples, and the antibody solution [anti-proNT/NMN rabbit polyclonal antibody (105S), 1/3,000 dilution] were added and incubated with shaking for $3 \mathrm{~h}$ at room temperature. Plates were washed with PBS containing $0.05 \%$ Tween-20 four times before the addition of labeling solution (streptavidin, horseradish peroxidase conjugate; Merck, Darmstadt, Germany), and incubated for $2 \mathrm{~h}$ at room temperature. After four final washes, BM chemiluminescence ELISA substrate (POD) solution (Roche Applied Science, Mannheim, Germany) was added to each well and allowed to develop in the dark for $3 \mathrm{~min}$. The chemiluminescence of BM ELISA was measured using Wallac 1420 ARVO MX (Perkin-Elmer Life Sciences, Waltham, MA, USA). A standard curve for proNT/NMN levels was obtained using proNT/NMN (aa 40-87) as the standard antigen.

Immunoblotting. Proteins were separated by SDS-PAGE on $14.25 \%$ polyacrylamide gels and electrophoretically transferred onto polyvinylidene difluoride (PVDF) membranes (Millipore, Bedford, MA, USA). Immunoblotting was performed using anti-proNT/NMN rabbit polyclonal antibodies (105S, 1:1,000 dilution), or anti-neurotensin goat polyclonal antibodies (N-17 and C-19, 1:1,000 dilution; Santa Cruz Biotechnology Inc., Santa Cruz, CA, USA) as the primary antibody, and anti-rabbit IgG-, or anti-goat IgG-horseradish peroxidase (HRP) conjugate (1:10,000 dilution; Jackson ImmunoResearch, West Grove, PA, USA) as the secondary antibody. HRP-dependent luminescence was developed using ECL Prime Western blotting detection reagent (GE Healthcare UK Ltd., Buckinghamshire, UK) and detected in a LAS-3000 (Fuji Film, Tokyo, Japan). 
A

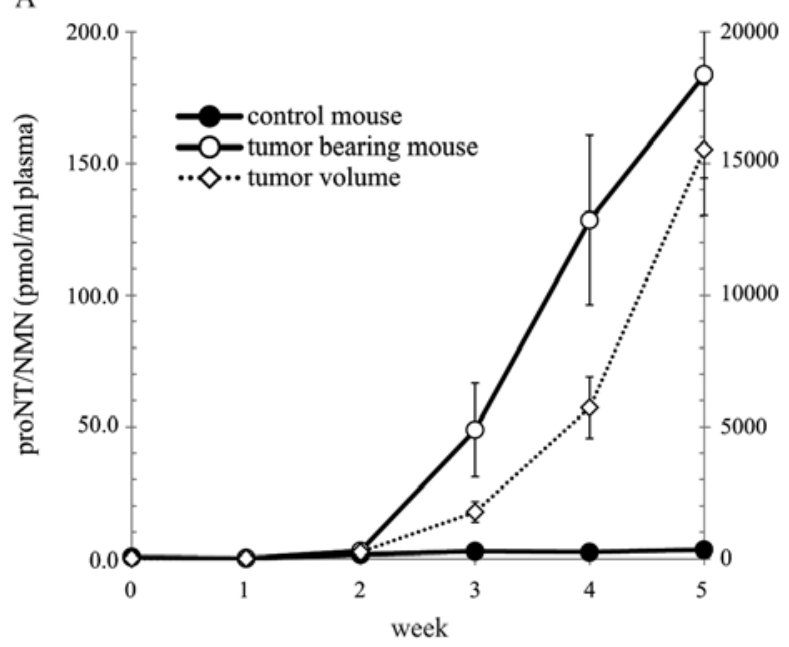

B

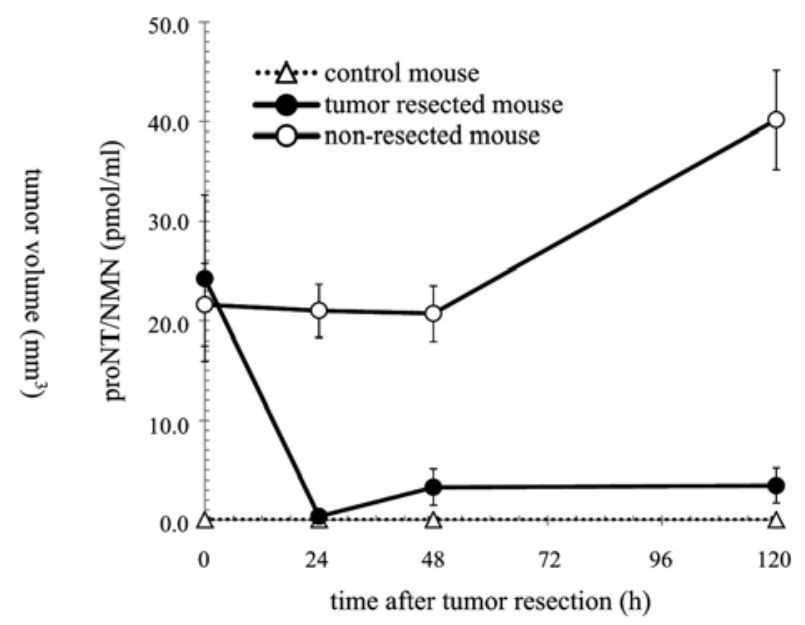

Figure 2. Time course of proNT/NMN levels in the plasma (A) and the effect of tumor resection on proNT/NMN levels (B). (A) Plasma was collected from nude mice at $0,1,2,3,4$ and 5 weeks after inoculation. ProNT/NMN levels in the plasma [control mouse $(\bullet)$ and tumor-bearing mouse $(\circ)$ ] were measured by ELISA, as described in Materials and methods. The tumor volume $(\diamond)$ was calculated using the formula: tumor volume $\left(\mathrm{mm}^{3}\right)=\left(\right.$ length $\mathrm{x}$ width $\left.{ }^{2}\right) / 2$. $(\mathrm{B}) \mathrm{Four}$ weeks after inoculation, tumors were resected from the NRG mice, and plasma samples were collected from all groups of mice at $0,24,48$ and $120 \mathrm{~h}$ after tumor resection. ProNT/NMN levels in the plasma [control mouse $(\Delta)$, tumor-resected mouse $(\bullet)$ and non-resected mouse ( $\circ)$ ] were measured by ELISA, as described in Materials and methods. Data are expressed as the means \pm SE values in multi-replicate experiments ( $\mathrm{n}=4-5)$. Statistical significance $\left({ }^{*} \mathrm{P}<0.05\right.$ as compared with the group of non-resected mouse) was evaluated by Student's t-test.

Immunohistochemistry. Formalin-fixed tumor tissues were gradually dehydrated in ethanol and embedded in paraffin. Tissue sections (3- $\mu \mathrm{m}$ thick) were cut and subjected to immunohistochemistry. Sections were deparaffinized in xylene, and treated with $1 \% \mathrm{H}_{2} \mathrm{O}_{2}$ in methanol for $30 \mathrm{~min}$ to block the endogenous peroxidase activity. After blocking, they were incubated with anti-proNT/NMN polyclonal antibody (1:200 dilution) or with the antibody in the presence of the antigen peptide for $30 \mathrm{~min}$ at room temperature. The sections were then washed with PBS and incubated with peroxidase labeled polymer (goat anti-rabbit IgG; Dako, Glostrup, Denmark) for 30 min. Following one wash with PBS, peroxidase activity was initiated with DAB substrate solution (Dako). The peroxidase reaction was completed, the sections were washed with PBS, counterstained with hematoxylin, dehydrated, cleared in xylene, and a coverslip was placed over the sections.

Statistical analysis. The results of the experiments are expressed as the mean \pm SE values. The statistical significance of differences was determined according to the Student's t-test.

\section{Results}

Evaluation of the proNT/NMN levels in tumor-bearing mice. We first established a rabbit polyclonal antibody against the proNT/NMN (aa 8-87) and developed a competitive ELISA system. Based on the results of the standard curve with proNT/NMN (aa 40-87) (Fig. 1B), the proNT/NMN levels in mouse plasma were measured by ELISA. Every week, plasma samples were collected from the control and SBC3-inoculated mice after inoculation. As shown in Fig. 2A, tumor volumes of SBC3-inoculated mouse gradually increased. Correlating with tumor volumes, the plasma proNT/NMN levels of the tumorbearing mice were elevated, while the proNT/NMN levels in control mouse were not significantly detectable.
To further investigate proNT/NMN secretion, tumors were surgically resected from the NRG mice intradermally inoculated with SBC3 cells, and the plasma proNT/NMN levels were evaluated after resection. Compared to nude mice that were subcutaneously inoculated with SBC3 cells, the tumors of NRG mice grew slowly, and the plasma proNT/NMN levels were slightly elevated 4 weeks after inoculation (nude mice, $128.49 \pm 32.18 \mathrm{pmol} / \mathrm{ml}$ plasma; NRG mice, $22.93 \pm 4.67 \mathrm{pmol} /$ $\mathrm{ml}$ plasma). The differences in the plasma proNT/NMN levels between the control and intradermally inoculated NRG mice were statistically significant. After surgical resection, the plasma proNT/NMN levels of the tumor-resected mice decreased until they reached the level identical to that of the control mice (Fig. 2B). Although the levels of the tumorbearing mice (non-resected mice) were significantly elevated after $120 \mathrm{~h}$, the levels of the tumor-resected mice remained the same as that of the control mice. These results clearly suggest that SBC3 tumors secreted proNT/NMN into blood.

Immunoblot detection of proNT/NMN in mouse plasma. Plasma proNT/NMN was also detected by immunoblotting using the same antibody as used in the ELISA. In SBC3 serum-free conditioned medium, the positive band was detected around $17 \mathrm{kDa}$ (Fig. 3A left line, SBC3-CM). The medium contains proNT/NMN (14), and the theoretical molecular mass of proNT/NMN is about $17 \mathrm{kDa}$, suggesting that the band detected around $17 \mathrm{kDa}$ is specific to proNT/ NMN. In mouse plasma, several bands were detected around the molecular mass of $17 \mathrm{kDa}$. The band at $17 \mathrm{kDa}$, specific to full-length proNT/NMN, was only detected in tumor-bearing mouse plasma; however, the lower bands ( 14-16 kDa) were detected in the plasma samples from both the tumor-bearing and control mice (Fig. 3A).

To gain more insight into whether the lower bands are derived from proNT/NMN, immunoblotting was performed 
A
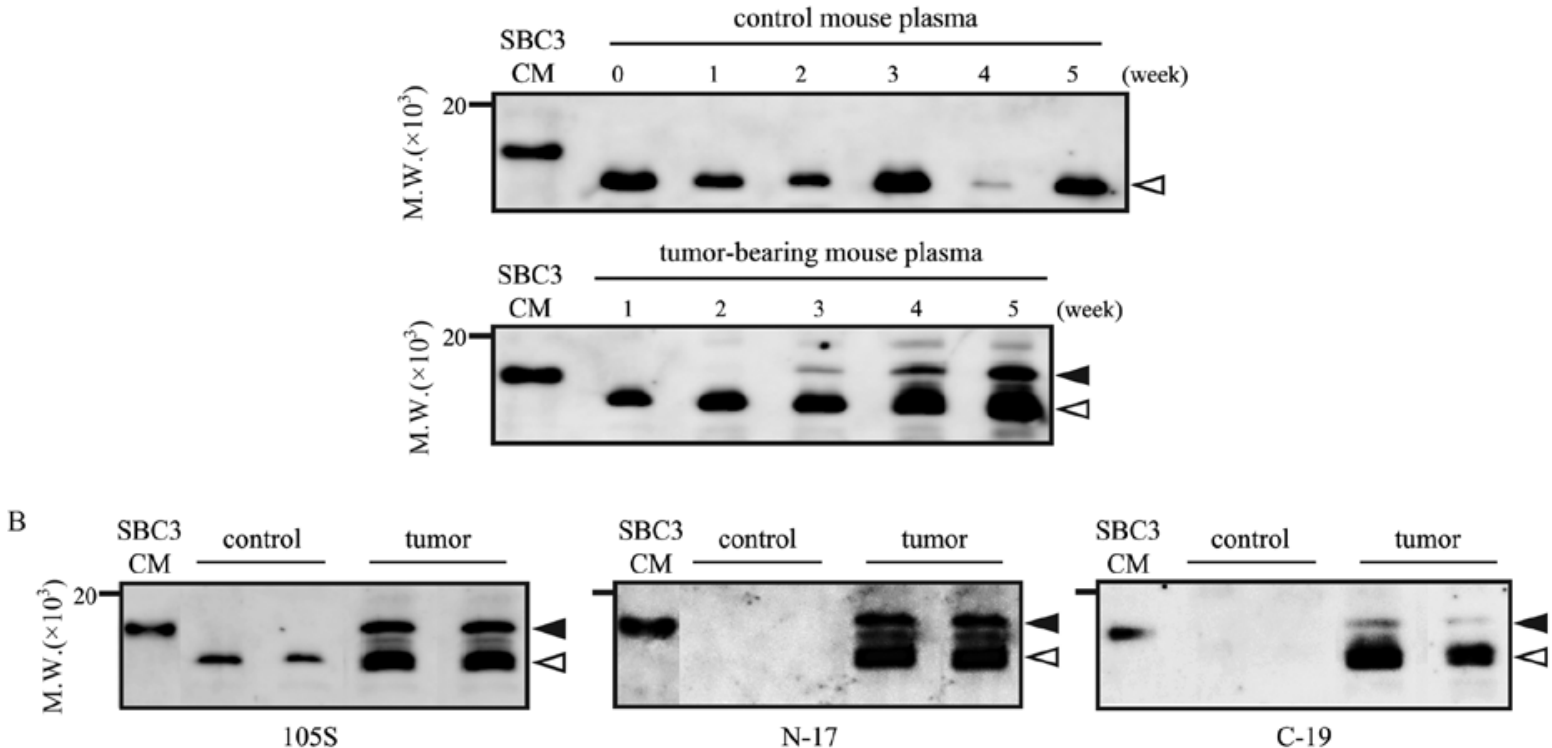

Figure 3. Immunoblot detection in mouse plasma. (A) ProNT/NMN levels in the mouse plasma (upper panel, control mouse; lower panel, tumor-bearing mouse) was determined by immunoblotting. SBC3 serum-free conditioned medium $(0.25 \mu 1 /$ lane, indicated as SBC3 CM) and plasma samples $(1 \mu 1 /$ lane $)$ were separated on $14.25 \%$ polyacrylamide gels, and electrophoretically transferred onto PVDF membrane. ProNT/NMN was detected by anti-proNT/NMN rabbit polyclonal antibody, as described in Materials and methods. (B) SBC3 serum-free conditioned medium (SBC3 CM) and proNT/NMN in the mouse plasma (control, control mouse plasma; tumor, tumor-bearing mouse plasma) were also detected by immunoblotting using anti-NT antibodies (N-17, C-19), as described in Materials and methods.

A

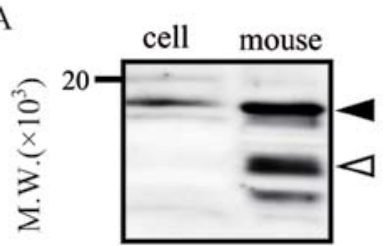

B
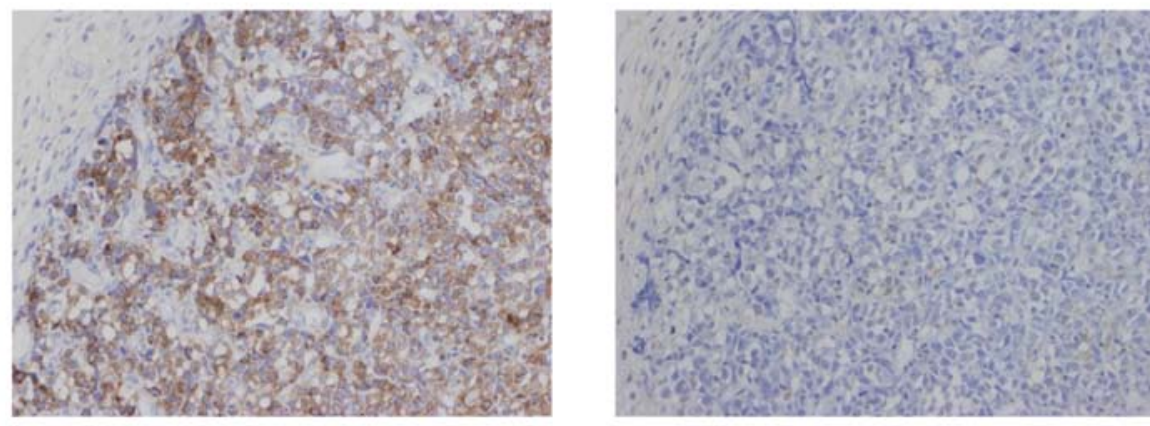

+peptide

Figure 4. ProNT/NMN expression in mouse tumor tissues. (A) SBC3 cell lysates (20 $\mu \mathrm{g} /$ lane, indicated as cell) and mouse tumor tissue lysates (10 $\mu \mathrm{g} / \mathrm{lane}$, indicated as mouse) were used for immunoblotting, as described above. (B) Immunohistochemical analyses were performed in mouse tumor tissues, as described in Materials and methods. ProNT/NMN was stained with DAB (brown color), and the nuclei were counterstained with hematoxylin (purple color). Tissue sections were incubated with anti-proNT/NMN polyclonal antibody (left panel) or incubated with the antibody in the presence of proNT/NMN (aa 8-87) (right panel).

using anti-NT antibodies, N-17 and C-19. Both antibodies can recognize NT-containing products such as NT, large NT, proNT/NMN, and N-17 can also recognize large NMN. Using these two antibodies, the bands around 14-16 kDa were detected in the plasma of tumor-bearing mouse, but not in the plasma from the control mouse (Fig. 3B). These results suggest that the lower band detected in the control mouse plasma is not derived from secreted proNT/NMN, but in the tumor-bearing mouse plasma, the processing products of proNT/NMN such as large NMN and large NT, were detected around 14-16 kDa.

ProNT/NMN expression in tumor tissues. ProNT/NMN expression in mouse tumor tissues was also determined by immunoblotting and immunohistochemistry. As with the 
plasma samples, the positive bands derived from proNT/NMN were detected in the SBC3 tumor tissues (Fig. 4A). In addition, Fig. 4B shows that proNT/NMN was expressed particularly in the superficial layer of the tumor (left panel). Immunostaining was diminished when the tumor tissue section was treated with antibody in the presence of the antigen peptide (right panel). This peptide competition analysis confirmed the expression of proNT/NMN in the above-mentioned areas in the tumor tissue. ProNT/NMN expression in the tumor tissues indicated that this precursor was produced by SBC3 tumors and secreted into plasma as the tumors grew.

\section{Discussion}

Most peptide hormones including neuropeptides are derived from the processing of larger inactive polypeptide precursors by prohormone convertases (PCs). PC-mediated processing often generates different combinations of active peptides. $\mathrm{NT}$ is derived from the large precursor proNT/NMN and this precursor is mainly processed by PC1, PC2 and PC5 $(8,15)$. Each $\mathrm{PC}$ cleaves at a different site of proNT/NMN; therefore, the pattern of processing products depends on the $\mathrm{PC}$ being expressed. Previous studies have demonstrated that the major products derived from proNT/NMN are NMN, NT, and large $\mathrm{NT}$ in the brain, large NMN in the gut, and NT, large NMN, and large NT in the adrenals $(4,16-18)$. Moreover, proNT/NMN processing occurs in only $\sim 50 \%$ of a subset of colon cancer cell lines, because $\mathrm{PC} 1$ and $\mathrm{PC} 2$, which are mainly related to the proNT/NMN processing, are not expressed in these cell lines (3). Most SCLC cell lines, including SBC3, do not concurrently express $\mathrm{PC} 1, \mathrm{PC} 2$ and $\mathrm{PC} 5$ (data not shown). Only the precursor was detected in the SBC3 cells and the conditioned medium, whereas the large forms of processing products were not detected (Fig. 3). On the other hand, not only proNT/ $\mathrm{NMN}$, but also the large processing products, was detected in the plasma of SBC3 tumor-bearing mice. These results suggest that proNT/NMN was the major product secreted from SCLC and the large forms of processing products were also produced in the SCLC tumor-bearing mice.

Neuropeptides are generally easy to metabolize, and therefore, have a short half-life in blood. Gastrin-releasing peptide (GRP) is one of the prototypical neuropeptide hormones. Although GRP is produced in high amounts and secreted into blood by SCLC cells, the unstable nature renders its detection in blood quite difficult. Therefore, the ProGRP (aa 31-98), which is also secreted into blood, was established as a useful tumor marker for SCLC (19-21). NT is also known to be more unstable than its precursor in vivo $(22,23)$, as well as in vitro (13). While the production and secretion of $\mathrm{NT}$, as well as the expression of NT receptors, were detected in many lung cancer cell lines including SCLC and NSCLC (24-26), it remains unclear whether NT or NT receptors are potential useful tumor markers. In fact, the plasma NT levels in the SBC3 tumor-bearing mice were slightly elevated compared with the control mice and were detected in the tumors. However, the NT levels were much lower than the proNT/NMN levels (data not shown). To our knowledge, this is the first report to investigate proNT/NMN secretion from SCLC tumors and to evaluate the plasma levels of proNT/ NMN itself. Our findings suggest that proNT/NMN might be a potential tumor marker for SCLC. To confirm this possibility, further analysis of the blood samples from SCLC patients is required.

\section{Acknowledgements}

This research was partially supported by the Ministry of Education, Science, Sports and Culture, grant-in-cooperation of the Regional Innovation Cluster Program 2010. The authors thank T. Ide for kindly providing technical assistance.

\section{References}

1. Kislauskis E, Bullock B, McNeil S and Dobner PR: The rat gene encoding neurotensin and neuromedin N. Structure, tissuespecific expression, and evolution of exon sequences. J Biol Chem 263: 4963-4968, 1988.

2. Carraway RE, Mitra SP, Feurle GE and Hacki WH: Presence of neurotensin and neuromedin-N within a common precursor from a human pancreatic neuroendocrine tumor. J Clin Endocrinol Metab 66: 1323-1328, 1988

3. Rovere C, Barbero P, Maoret JJ, Laburthe M and Kitabgi P: Pro-neurotensin/neuromedin $\mathrm{N}$ expression and processing in human colon cancer cell lines. Biochem Biophys Res Commun 246: 155-159, 1998.

4. Carraway RE, Mitra SP and Spaulding G: Posttranslational processing of the neurotensin/neuromedin-N precursor. Ann N Y Acad Sci 668: 1-16, 1992.

5. Carraway RE, Mitra SP and Muraki K: Isolation and structures of xenopsin-related peptides from rat stomach, liver and brain. Regul Pept 29: 229-239, 1990.

6. Bidard JN, de Nadai F, Rovere C, et al: Immunological and biochemical characterization of processing products from the neurotensin/neuromedin $\mathrm{N}$ precursor in the rat medullary thyroid carcinoma 6-23 cell line. Biochem J 291: 225-233, 1993.

7. Rovere C, Barbero P and Kitabgi P: Evidence that PC2 is the endogenous pro-neurotensin convertase in rMTC 6-23 cells and that PC1- and PC2-transfected PC12 cells differentially process pro-neurotensin. J Biol Chem 271: 11368-11375, 1996.

8. Kitabgi P: Differential processing of pro-neurotensin/neuromedin $\mathrm{N}$ and relationship to pro-hormone convertases. Peptides 27: 2508-2514, 2006.

9. Zhao D and Pothoulakis C: Effects of NT on gastrointestinal motility and secretion, and role in intestinal inflammation. Peptides 27: 2434-2444, 2006.

10. Davis TP, Burgess HS, Crowell S, Moody TW, CullingBerglund A and Liu RH: Beta-endorphin and neurotensin stimulate in vitro clonal growth of human SCLC cells. Eur J Pharmacol 161: 283-285, 1989.

11. Tallett A, Chilvers ER, MacKinnon AC, Haslett $\mathrm{C}$ and Sethi T: Neuropeptides stimulate tyrosine phosphorylation and tyrosine kinase activity in small cell lung cancer cell lines. Peptides 17: 665-673, 1996.

12. Moody TW, Chiles J, Casibang M, Moody E, Chan D and Davis TP: SR48692 is a neurotensin receptor antagonist which inhibits the growth of small cell lung cancer cells. Peptides 22: 109-115, 2001.

13. Friry C, Feliciangeli S, Richard F, Kitabgi P and Rovere C: Production of recombinant large proneurotensin/neuromedin $\mathrm{N}$-derived peptides and characterization of their binding and biological activity. Biochem Biophys Res Commun 290: 1161-1168, 2002.

14. Ogura S, Kaneko K, Miyajima S, Ohshima K, Yamaguchi K and Mochizuki T: Proneurotensin/neuromedin N secreted from small cell lung carcinoma cell lines as a potential tumor marker. Proteomics Clin Appl 2: 1620-1627, 2008.

15. Villeneuve P, Feliciangeli S, Croissandeau G, et al: Altered processing of the neurotensin/neuromedin $\mathrm{N}$ precursor in $\mathrm{PC} 2$ knock down mice: a biochemical and immunohistochemical study. J Neurochem 82: 783-793, 2002.

16. Shaw C, McKay D, Johnston CF, et al: Differential processing of the neurotensin/neuromedin $\mathrm{N}$ precursor in the mouse. Peptides 11: 227-235, 1990.

17. Carraway RE, Mitra SP and Paradise C: Characterization of large neuromedin- $\mathrm{N}$ using antisera towards regions of the neurotensin/ neuromedin-N precursor. Peptides 12: 601-607, 1991. 
18. de Nadai F, Rovere C, Bidard JN, Cuber JC, Beaudet A and Kitabgi P: Post-translational processing of the neurotensin/ neuromedin $\mathrm{N}$ precursor in the central nervous system of the rat. I. Biochemical characterization of maturation products. Neuroscience 60: 159-166, 1994.

19. Yamaguchi K, Abe K, Kameya T, et al: Production and molecular size heterogeneity of immunoreactive gastrin-releasing peptide in fetal and adult lungs and primary lung tumors. Cancer Res 43: 3932-3939, 1983.

20. Miyake Y, Kodama T and Yamaguchi K: Pro-gastrin-releasing peptide(31-98) is a specific tumor marker in patients with small cell lung carcinoma. Cancer Res 54: 2136-2140, 1994.

21. Stieber P, Dienemann H, Schalhorn A, et al: Pro-gastrinreleasing peptide (ProGRP) - a useful marker in small cell lung carcinomas. Anticancer Res 19: 2673-2678, 1999.
22. Barelli H, Fox-Threlkeld JE, Dive V, Daniel EE, Vincent JP and Checler F: Role of endopeptidase 3.4.24.16 in the catabolism of neurotensin, in vivo, in the vascularly perfused dog ileum. Br J Pharmacol 112: 127-132, 1994.

23. Checler F, Amar S, Kitabgi P and Vincent JP: Catabolism of neurotensin by neural (neuroblastoma clone N1E115) and extraneural (HT29) cell lines. Peptides 7: 1071-1077, 1986.

24. Moody TW, Carney DN, Korman LY, Gazdar AF and Minna JD Neurotensin is produced by and secreted from classic small cell lung cancer cells. Life Sci 36: 1727-1732, 1985.

25. Moody TW: Peptide hormones and lung cancer. Panminerva Med 48: 19-26, 2006.

26. Alifano M, Souaze F, Dupouy S, et al: Neurotensin receptor 1 determines the outcome of non-small cell lung cancer. Clin Cancer Res 16: 4401-4410, 2010. 